\title{
Knowledge base Construction using Hidden Web Retrieval Technique
}

\author{
Shrina Patel \\ $U$ and P.U. Patel Department of Computer \\ Engineering, \\ Chandubhai S Patel Institute of Technology, \\ Charotar University of Science \\ and Technology, Changa-388421, Gujarat, India
}

\author{
Amit Ganatar \\ $U$ and P.U. Patel Department of Computer \\ Engineering, \\ Chandubhai S Patel Institute of Technology, \\ Charotar University of Science \\ and Technology, Changa-388421, Gujarat, India
}

\begin{abstract}
Relations of algorithms for hidden web-focused information retrieval develop with it. When the stage the information retrieval, a huge difficulty arises and that is the information that one hidden web page can enclose manifold areas with extremely dissimilar information content. Hence the page has to be split into measurement and these parts examined separately for the results to be more precise. Web page segmentation and correlated technique have Therefore this paper suggests a combined approach that creates use of structural features and the visual features. It build a visual DOM tree on which the data records are recognized based on their structural similarity. The structure of these data records are reserved so that personage data items can be group effortlessly and precisely based on their visual features Which hidden web source do we intend at the information indispensable to access the data at the back web form and the type of interface. We proposed algorithm narrative vision based page segmentation (NVIPS) and also comparison DOM tree, VIPS
\end{abstract}

\section{Keywords}

Hidden web crawling, web database, DOM tree, VIPS, VIPS.

\section{INTRODUCTION}

Centralized search engines like yahoo, Bing and Google use crawlers to download web content and build an . Inverted index so that users can quickly search within the content. Crawlers are specified a set of seed pages and recursively download content by subsequent the links on the downloaded pages. However, many pages.

This content is frequently of high quality and highly relevant to the user's information require. In other words, it is of crucial importance to provide adequate hidden web search functionality. For the remainder of this proposal, the inaccessibility of hidden web pages to web crawlers will be referred to as the hidden problem.

A different problem related to web search is its immense size and continuous growth, which poses a lot of challenge and hard requirements on the scalability of several web search solution [6].In 1999, it was probable that no web search engine indexes more than $16 \%$ of the surface web, and that the web consisted of 800 million pages [10]. In 2005, a novel estimate put this number at 11.5 billion pages [11], and in 2008, Google announced the discovery of one trillion unique URLs on the web at once. In 2010 Understanding Deep Web Search Interfaces[13]. in 2011 Efficient Maintenance of Common Keys in Archives of Continuous Query Results from Deep Websites[14]. in 2012 Automated Form Understanding for the Deep Web[15]. In 2014 unlimited URLs on the web at once the extremely large number of web pages and the continuous web growth will be referred to as the big problem.
A key effort of, integrating, retrieve and mining successful and raised dominance information from huge hidden web knowledge online is how to recurrently and efficiently conclude and identify domain exact hidden web knowledge entry points, searchable forms, in the Web.

In this research, to recommend a new technique of a smart agent based crawler for domain-specific Hidden Web databases has been recommend attending to the limitations of the obtainable method. Combined approach that creates use of structural features and the visual features. It build a visual DOM tree on which the data records are recognized based on their structural similarity Our work addresses the greatest issue of vision-based page segmentation and that is time complexity. Our approach is faster than the vision-based page segmentation while keeping the same level of accuracy. The method, called Cluster based Page Segmentation, builds on existing algorithms but it is not strictly bound to any particular one of them any vision-based page segmentation algorithm can be used if its implementation follows some basic rules. It is not even strictly bound to vision-based segmentation although it has the greatest potential when used with it. From this perspective, the Cluster-base Page Segmentation is a complementary algorithm. It is based on templates, a principle on which modern web pages greatly depend. When we consider the principle of templates, it is possible to group pages from the same site in several clusters. Then we can create cluster-bound structures containing information common to all pages in their respective clusters. These structures are called Cluster Representatives. Each of these representatives contains information about DOM tree of its cluster, meaning it can be segmented. From that point the segmentation of all other pages is unnecessary because each Cluster Representative corresponds to every page in its cluster.

Besides that this paper algorithms for utilizing these structures. The maintenance eon smart knowledge agent and domain ontology, and a classification of narrative and efficient strategies, including a two-step page classifier, an association scoring approach, etc, it can get improved the performance of the obtainable technique. We accomplish number of real hidden Web pages in a set of representative domains conduct and the significances establish that the in terms of the harvest rate, attention rate and time performance.

The greatest important motive on accessing hidden web data. However, access supplementary data sources is not the just cause which kinds hidden web data stimulating for users, companies and consequently for researchers. In subsequent, a number of extra reasons are mention to authorize the challenge for access hidden web data.

Therefore certain technique or tool is essential for retrieving such enormous volume of information. The difficulties with 
the conservative search engines can be not clever to index hidden web, deficiency of personalization, consequences with low precision and recall and not able to comprise user feedbacks founded on specific domain [3].

Alternative significant difficult is to discovery out and classify the access points of the hidden web databases i.e. form, in the web efficiently and automatically. The following factors are responsible for the complication of the above problems [4] The size of hidden web databases is in large amount with the continuous increase in growth of web databases sites.

It is problematic to different iateamong searchable forms and non-searchable forms. To resolve the exceeding revealed difficulties efforts have been completed. But the current resolutions are not clever to resolve the problem entirely. Consequently to resolve the difficulty of discovery out and classifying the web databases form automatically the existing solutions are desirable to be protracted extra to proliferation both the precision and recall.

This can be done by retrieving the more and more relevant documents from domain-specific hidden web databases. For this, numerous methods have been projected previous but have some limitations. The newest effort done is identified as improved form intensive crawler. For innovative effort an smart agent technology is familiarized in that work for retrieving supplementary and more relevant information in directive to growth both precision and recall.

\section{RELATED AND COMPARATIVE WORKS}

Foremost search engines every were able to index fragment of the hidden web. Though, practically two thirds of the hidden web was not indexed by any engine, representative convinced characteristic barriers for crawling and indexing the hidden web. Present methods connected to searching hidden content comprise universal search of enterprise verticals domain precise mediators like and surfacing i.e. automatically filling in and submitting web forms, and indexing the resulting web pages. Many researcher work in this domain.

Chelsea Hicks in at al[1] With further and more evidence goes online, extracting and supervision the information from the www is attractive progressively important. Though the surface Web's information is comparatively easy to get thanks to search engines such as Google and Bing, gathering the information from the hidden Web is stagnant a stimulating task and these search engines do not index information located inside the hidden Web. Associated to the surface Web, the hidden Web encompasses enormous more information.

Y. Li, Y. Wang and J. Du[2]in this research, an enhanced form-focused crawler for domain-specific wdbs (e-ffc) has been proposed as a novel framework to address current solutions' restrictions. the e-ffc, founded on the divide and conquer strategy, employs a series of original and active strategy algorithms, include a two-step page classifier, a link score approach, classifiers for progressive searchable and domain-specific forms, crawling stopping standards, etc.

Q. Huanga, in at al[3]proposed an effective and efficient method is proposed to resolve this difficult. In the method, a set covering model is used to designate the web database based on this model an incremental harvest model is learned by the machine learning technique to choice the suitable query automatically.

K. K. Bhatia, in at al[4]In this work, they have design of a domain-specific hidden web crawler is being planned that automates the form filling procedure to allow crawling of the hidden web. The tests showed on Domain-specific Hidden Web Crawler (AKSHR) designate that it professionally crawls the hidden web pages.

Madhavanet. al. [5] described the technical revolutions fundamental the main large-scale Deep-Web surfacing system. The consequences or our surfacing are presently liked by millions of users per day internet, and envelop content in over 700 domains, over 50 languages, and beginning a number of million forms. The influence on search traffic is a substantial authentication of the importance of Deep-Web content. Methods: Input values for text search inputs based approach, Strength: It can efficiently navigate for searching against various possible input combinations, User's participation: no, Automatic query assortment: yes, Absorbed crawling: no, Precision: average, Recall: average, Limitations: Problem in forms associated with java script.

Sergio Flesca in at al[6]propose an algorithm for wrapper evaluation that works in polynomial time with respect to the size of a PDF document, being parametric to a truth value threshold that fuzzily controls the group expansion. Effectiveness and efficiency of our PDF wrapping approach have been assessed their work was not good accuracy in wrapping real-world PDF documents that exhibit different characteristics and come from various domains such as, e.g., balance sheets, newspapers and magazines, data and time sheets, price lists, weather forecast reports.

Jer Lang Hong in at al[7]The ontological technique could also reduce the number of potential data regions for data extraction and this was shorten the time and increase the accuracy in identifying the correct data region to be extracted. Measurement of the size of text and image to locate and extract the relevant data region further improves the precision of our wrapper.

Gang Liu in at al[8]Hidden Web data sources found that the problem has been difficult in the study of Hidden Web. This paper based on the topic crawler joined the Hidden Web entry found module, to automatically discovered Hidden Web entry interface of related domain. However, use the entry detection module, it is able to check the collected Hidden Web entry pages, and remove irrelevant pages to ensure the accuracy of Hidden Web data sources.

Barbosa et. al [9]proposed solution called Hidden Peep that uses a hidden-web crawler which is a focused-crawler for sparse concept on the web, a clustering algorithm for organizing a large set of forms, and an ensemble learning technique to automatically extract labels from discovered hidden web forms and index them. HiddenPeep can be found online and works for multiple domains. 
Table 1: Comparative analysis Different Works

\begin{tabular}{|c|c|c|c|c|}
\hline Topics & Algorithm & Strength & Limitations & Authors \\
\hline $\begin{array}{l}\text { Towards Web-Scale } \\
\text { Structured Web Data } \\
\text { Extraction }\end{array}$ & $\begin{array}{l}\text { Record Segmentation } \\
\text { and Extraction }\end{array}$ & $\begin{array}{l}\text { proposed method to be } \\
\text { deployed in Web-Scale } \\
\text { structured data } \\
\text { extraction system }\end{array}$ & $\begin{array}{l}\text { Not working well adding } \\
\text { visual feedback for } \\
\text { correcting errors }\end{array}$ & Tomas Grigalis[2013] \\
\hline $\begin{array}{l}\text { A New Architecture } \\
\text { of an Intelligent } \\
\text { Agent-Based } \\
\text { Crawler for Domain- } \\
\text { Specific Deep } \\
\text { Web Databases }\end{array}$ & $\begin{array}{l}\text { Deep Web } \begin{array}{r}\text { Form- } \\
\text { Focused } \\
\text { (FFCs) }\end{array} \\
\text { Crawlers }\end{array}$ & $\begin{array}{l}\text { Due to crawler } \\
\text { generated query } \\
\text { crawling is efficient }\end{array}$ & $\begin{array}{l}\begin{array}{l}\text { Does not focus on } \\
\text { frequently }\end{array} \\
\begin{array}{l}\text { used } \\
\text { database }\end{array}\end{array}$ & $\begin{array}{llr}\text { Yanni } & \text { LI, } & \text { Yuping } \\
\text { WANG , } & \text { Erfang } & \text { TIAN } \\
{[2012]} & & \end{array}$ \\
\hline $\begin{array}{lr}\text { Automated } & \text { Form } \\
\text { Understanding } & \text { for } \\
\text { the Deep We } & \end{array}$ & $\begin{array}{l}\text { OPAL(On-line } \\
\text { Information } \\
\text { Services) }\end{array}$ & $\begin{array}{l}\text { These two parts of } \\
\text { OPAL combined yield } \\
\text { form understanding } \\
\text { with } \\
\text { near perfect accuracy }\end{array}$ & $\begin{array}{l}\text { Retrieval of redundant } \\
\text { related information. }\end{array}$ & Tim Furche in at al[2012] \\
\hline $\begin{array}{l}\text { Clickstream } \\
\text { analysis and web } \\
\text { page text } \\
\text { similarity analysis } \\
\text { for parallel focused } \\
\text { crawler }\end{array}$ & $\begin{array}{l}\text { link based or context } \\
\text { based within a parallel } \\
\text { crawler }\end{array}$ & $\begin{array}{l}\text { Give more precise } \\
\text { answers set to } \\
\text { the users. }\end{array}$ & $\begin{array}{l}\text { User seesions are not } \\
\text { include }\end{array}$ & $\begin{array}{l}\text { Ahmadi-Abkenari, } \\
\text { F[2011] }\end{array}$ \\
\hline $\begin{array}{l}\text { A Novel Framework } \\
\text { for a Domain- } \\
\text { specific Hidden Web } \\
\text { Crawler }\end{array}$ & $\begin{array}{l}\text { Domainspecific } \\
\text { Hidden Web Crawler } \\
\text { (AKSHR) }\end{array}$ & 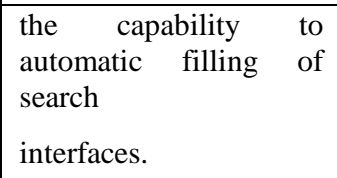 & $\begin{array}{l}\text { In this work cannot be done } \\
\text { in developing a specialized } \\
\text { search engine for Hidden } \\
\text { Web. }\end{array}$ & $\begin{array}{lrr}\text { Komal } & \text { Kumar } & \text { Bhatia, } \\
\text { A.K. Sharma, } & \text { Rosy } \\
\text { Madaan[2010] } & \end{array}$ \\
\hline $\begin{array}{l}\text { Understanding Deep } \\
\text { Web Search } \\
\text { Interfaces: A Survey }\end{array}$ & $\begin{array}{l}\text { 2-D } \\
\text { graph based algorithm }\end{array}$ & $\begin{array}{l}\text { formal study of the } \\
\text { correlation between the } \\
\text { extraction } \\
\text { methodologies and the } \\
\text { potential application } \\
\text { will be } \\
\text { greatly beneficial }\end{array}$ & $\begin{array}{l}\text { association among the } \\
\text { extraction } \\
\text { technique not applicable all } \\
\text { domain }\end{array}$ & $\begin{array}{l}\text { Ritu Khare, Yuan An, Il- } \\
\text { Yeol Song[2010] }\end{array}$ \\
\hline $\begin{array}{l}\text { Google'sDeepWeb } \\
\text { Crawl }\end{array}$ & $\begin{array}{l}\text { algorithm that } \\
\text { efficiently navigates } \\
\text { the search space of } \\
\text { possible input } \\
\text { combinations to } \\
\text { identify } \\
\text { only those that generate } \\
\text { URLs suitable for } \\
\text { inclusion into } \\
\text { our web search index. }\end{array}$ & $\begin{array}{lr}\text { It can efficiently } \\
\text { navigate for } & \\
\text { searching } & \text { against } \\
\text { various } & \\
\text { possible } & \text { input } \\
\text { combinations } & \\
\end{array}$ & $\begin{array}{l}\text { Handle forms powered by } \\
\text { Javascript and to consider } \\
\text { more carefully } \\
\text { dependencies between } \\
\text { values in different inputs of } \\
\text { a form }\end{array}$ & $\begin{array}{l}\text { Jayant Madhavan, David } \\
\text { Ko[2008] }\end{array}$ \\
\hline \multicolumn{5}{|c|}{$\begin{array}{ll}\text { 3. INFORMATION EXTRACTION } & \text { WISH algorithm: This technique for traversing the DOM tree } \\
\text { TECHNIQUE } & \begin{array}{l}\text { and choose applicable content. They objective so call data } \\
\text { proceedings which are pieces of a web page which are repeat } \\
\text { themselves, except with a dissimilar content. An example of }\end{array} \\
\text { 3.1 Dom Based Technique } & \text { such documentation can be a group or search consequences } \\
\text { The previous collection of segmentation methods is base on } & \begin{array}{l}\text { listing. Their algorithm is alienated in numerous steps. In the } \\
\text { initial step, they mine content contestant nodes using BFS- } \\
\text { common traversal of the DOM tree and recognizes the content } \\
\text { with usage of a variety of heuristics. Some of related works }\end{array} \\
\text { might not even be directly solving the segmentation by } & \begin{array}{l}\text { similar level of DOM tree, enclose recurring children } \\
\text { traversing the DOM tree. }\end{array}\end{array}$} \\
\hline
\end{tabular}


The parent is signify as data region. In case no nodes on a exacting level of BFS gratify the description, the next tree level is inspect. Output of the primary stage is a catalog of every one data regions recognized on the page. Other stages simply filter consequences the algorithm gained in the first phase. Following observations are used for filtering heuristics:

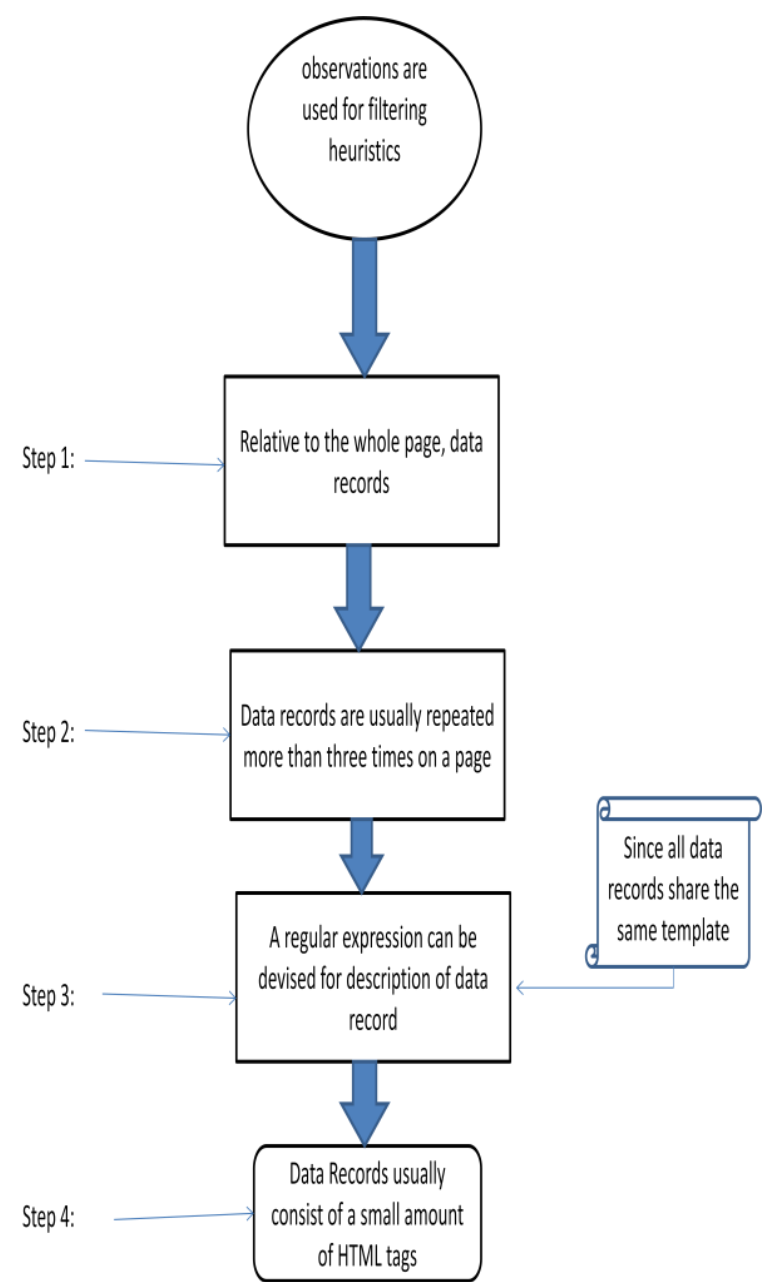

Figure 1: Dom Based Technique

Subsequent to the record of data regions is filtered all data region has to be assign its relevancy score. The categorization function illustrate determine the size of area in use by data records by including characters. Elements representing free space are taken into account as well. Data region with the best score is considered to be the main content of the page. This algorithm is the best example of how can dissimilar heuristics be use for page segmentation and classification.

\subsection{Narrative Visual Page Segmentation}

This relation of technique is base on an approach with effortless perception, except quite complex computing stress. The conception is to segment the web page as a customer would segment it if he was look at it. Vision -based page segmentation algorithm. VIPS is an essential algorithm in a family of visual based technique.

The algorithm uses one necessary period Degree of Coherence or merely DoC. It is a compute of visual coherence definite for every block. It can be symbolize by any numeral (integer or real), although it necessity produce with visual stability of the block. As well a parent can never have better DoC than its children in block hierarchy tree.

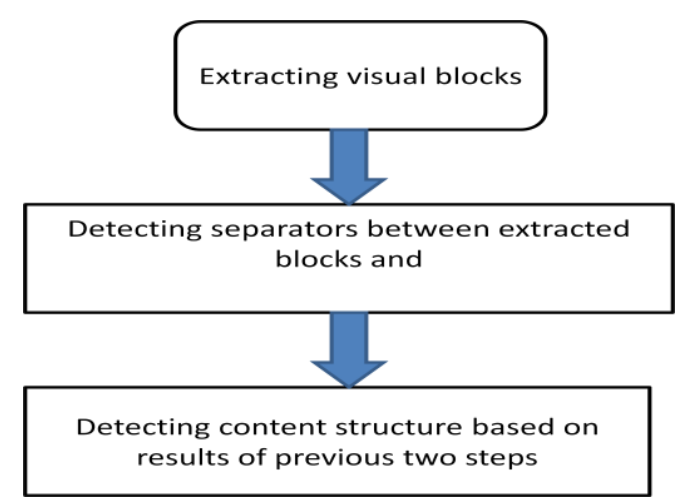

Figure 2: The algorithm segments page in three steps

Extracting visual blocks consists of a top-down tree walking throughout the DOM tree. The walk is iterative in every iteration a novel node in place of visual block is detected in the DOM tree. After this detection a decision is complete (base on convinced property similar to color, size, .) whether the block shall be recursively segmented added or not. For every detected block which isn't segmented more a DoC is set according to its visual coherence.

The subsequent step is partition detection. Separator is distinct as horizontal or perpendicular line or quite rectangular region which doesn't interconnect several of previously detected blocks. The algorithm is initialized by a particular separator covering the entire page. Separators are forever detected for a exacting stage of visual block tree. Then for every block we achieve a detection of its relative to every existing separator:

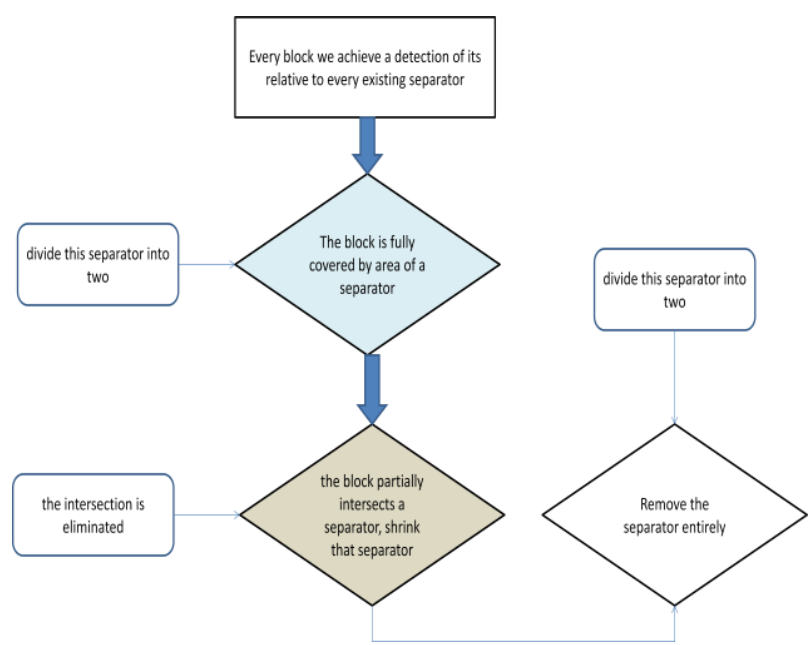

Figure 3: Narrative Visual Page Segmentation

The algorithm is ended by removing separators at the boundaries of document. After we have every separators, we allocate the weights base on visual dissimilarity of neighboring blocks. Note that this algorithm constructs either merely vertical or only horizontal separators. though the page has to be segmented in together directions. at this time it is significant to understand that the entire algorithm is done recursively.

That means that the page can be alienated merely in one direction, but some of its child nodes can be estranged on the other direction.

The concluding step of NVIPS is satisfied structure building. In this step we iterate all the way through a list of before found separators and combine visual blocks nearby to them. It's significant to merge blocks contiguous to separators with 
the negligible weight initial. previous to merging we have to ensure whether blocks get collectively granularity requirement. If they do, there is no require for merging them. If the block doesn't get together the prerequisite, we revisit to step one with root node organism that visual block.

This algorithm illustrates generally enhanced consequences than its predecessors, but it has a few shortcomings as well.

Phase 1: In a few cases straight division of a visual block is unfeasible and exploitation of virtual blocks is requisite. This can have negative impact on additional processing, since blocks are not actually present in the document.

Step2: consequential tree represent page segmentation but a number of information such as mutual point of blocks is missing. That information strength be useful for improving algorithm's outcome.

\subsection{Our Propose Algorithm and Basic Technique}

Hidden Web Source Discovery: In arrange to respond the information requirements of a user, it is essential to know from which data sources that information could be find. In the case of surface web, general search engines use the indexes and matching algorithms to locate those sources of interest. While in hidden web sources, the data is infertile behind web search forms and far from search engines attain. consequently, primary of all, it should be exposed that which hidden web data sources potentially enclose the data essential to respond a user query. To do so, the subsequent questions should be answered. How to conclude the probable hidden web sources for answer the query, taking into consideration the huge quantity of websites obtainable on the Web [6]

This could be complete by reduction down the search to find out hidden web sources of our concentration while having all the motivating hidden web sources covered.

A universal overview is provided on the suggested technique making data residing in hidden web sources obtainable to users.

Crawling replica:

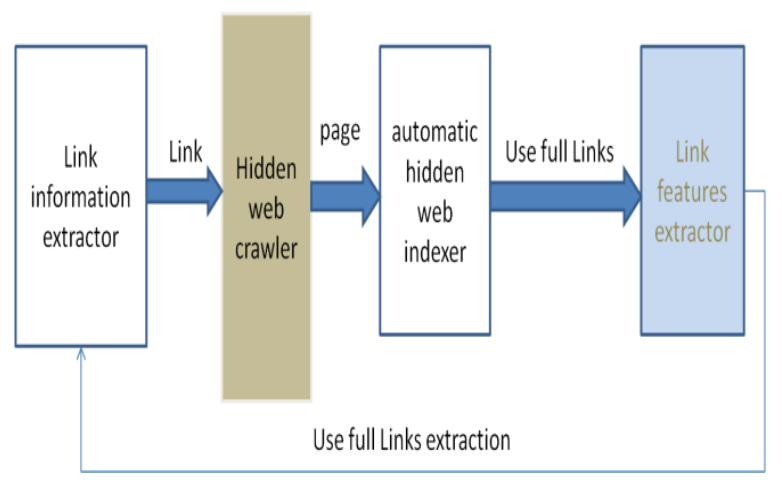

Figure 4: Hidden web extractor replica

Technique 1: providing Indexing authorization Data contributor permit product search services to index the data obtainable in their databases. This providea inclusive access to the data in the knowledge. Though, this approach is not appropriate in a spirited and unhelpful surroundings. In obstinate surroundings, the owners of a hidden website are unwilling to present any information which will be used by their competitors. For illustration, information about size and indexing algorithms, ranking, and underlying database features are denied to be accessed.
Technique 2: Crawling all Data obtainable in Hidden Web Repositories:This technique is base on the scheme of extract every one the data obtainable in hidden web repositories which are of users' interests and provide respond to their information requirements by affectation query on this extracted data [8]. This tolerates the web data source to be search and excavation in a federal approach. In regulate to extract data from hidden web data sources, their search form are used as the access points. Having filled in the input fields of these forms, the resultant pages are retrieved.

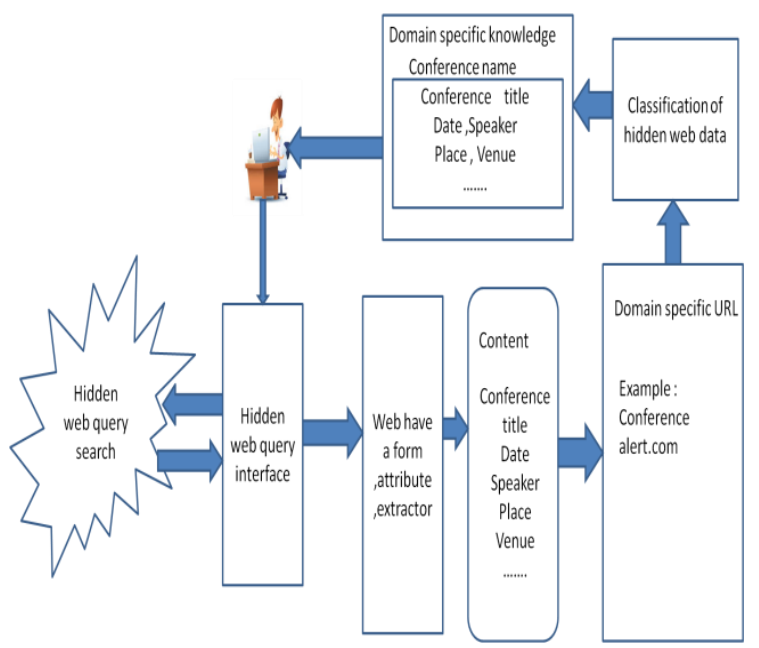

Figure 5: Propose system architecture

technique 3: Virtual Integration Heterogeneous of Search Engines In this technique of give data obtainable in hidden web repositories to users, mine every one the data beginning these sources is not besieged. as a substitute, it is tried to appreciate the forms give by dissimilar hidden data sources and give a matching mechanism which allow having one mediated form

Technique 4: developing Approach : In "Google's HiddenWeb Crawl by Carlos R. Osuna et al. [5], the objective is to obtain sufficient suitable example from every smart hidden web repository then the hidden web might have its accurate put in the consequences go back by search engines for the go through queries. This task is executed by precalculate the majority applicable capitulation for the HTML forms as the entry points to those hidden web repositories.

In our proposed technique uses smart agent knowledge for crawling information from hidden web. Two agents are used in the proposed work. One is for crawling website for extracting appropriate forms and the last synchronizes the outcomes from the crawling agents In every agent there are three modules. (a)is crawler which visits the web and transfers documents conferring to the query specified by the user. (b)Is classifier which has three sub- modules? Page classifier is recycled to regulate a page fits to which domain in the classification. Link classifier is used to discovery links with their features and methods which topics to pages that are targeted. Form classifier is used to distinguish between searchable form and non-searchable form and from them sieves out only searchable forms. The mined searchable forms are then examined to excellent those searchable forms which are in an absorbed domain and formerly they are supplementary to the database if they are not previously extant in the database. (c)Module is feature learner which studies pattern from database repeatedly to recover the presentation of all classifiers. Link classifier and form classifier, Page classifier. Out technique contains of smart 
agent controller which is used to regulate significance of link to be supervised. There are two agents which can achieve improved searching by examining and gathering information as feedback with the assistance of sharing previous crawling experience.

\section{EXPERIMENT RESULTS}

Step 1: appropriate to the heterogeneity of dissimilar conference Web pages, a number of rule-based Web information extraction techniques are not scalable some additional. The rules extract from one conference Web site can not be relevant to another conference, so we be supposed to discover out an technique self-sufficient starting page templates.

Step 2: A assortment of obtainable IE systems uses a DOM tree to stand for HTML page and complete information extraction base on the structure of the DOM tree. But HTML tags do not go after strict grammar confine; it is probable to source an error in parsing HTML DOM tree. In adding, DOM tree is to begin with designed to exhibit data in the browser, slightly than explain the semantic structure of Web pages, so still although two nodes have the equivalent parent node in the DOM tree, it does not mean they are added closely in semantic than additional nodes.

Step: 3 Traditional IR systems forever get a single Web page as input, except the constructive information of a conference can be positioned in manifold pages of the Web site, so the system be required to execute IR from portal level, and put together the extraction consequences of every page to absolute IR.

Primary experiment is verifying the complete tree. The vision tree outcome generate by VIPS and the absolute tree outcome generate by our new algorithm on 50 conference web portal. We can observe that the NVIPS trees have added leaf nodes than vision trees. It means our NVIPS can find more text blocks than VIPS.

The experimental consequences of removing noise blocks are known. We can observe some facts: there are a lot of noise blocks in the absolute tree. In several websites, approximately partially of every one blocks are noise blocks. Our eliminate noise method can remove average $42 \%$ noise nodes and $55 \%$ noise leaf nodes. consequently, it will diminish the number of nodes should be process in extraction and get better the efficiency.

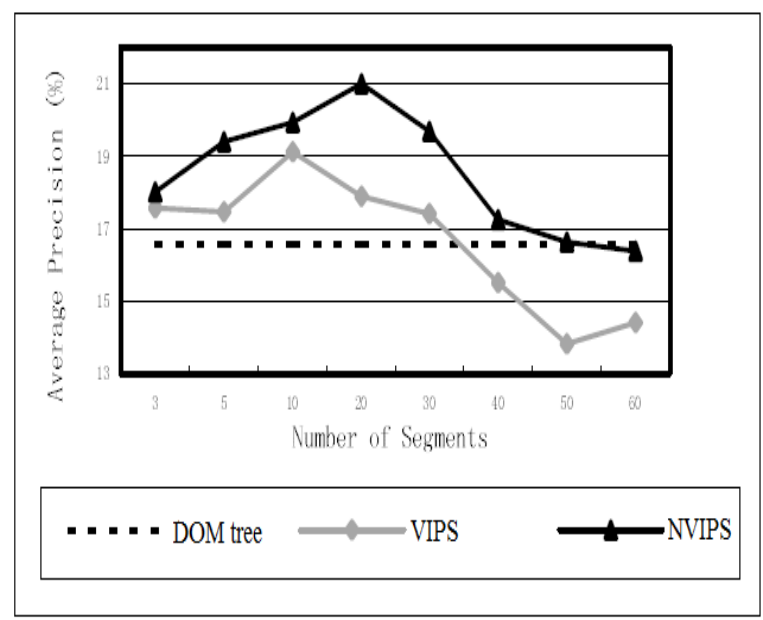

Figure 6: Performance comparison DOM tree, VIPS and NVIPS
The Figure 6 research is the comparison among initial categorization consequences and the outcome subsequent to post processing. The consequences are obtain on 25 arbitrarily websites. The preliminary classification outcome simply has average 0.76 precision, 0.68 recall and 0.69 F1- measure. subsequent to post-processing, the categorization consequences are enhanced to average 0.97 precision, 0.97 recall and 0.98 F1- measure. Consequently, the postprocessing key position in conference information extraction. a number of text blocks which have understandable vision and text content features, have improved classification outcome. The average F1-measure on these blocks is 0.99 .

\section{CONCLUSION}

Our propose research the structured data that is extracted can be used for processing in hidden web based applications in real time. The research effectively extracts the hidden web data records and data items using visual features. We create a database of hidden web pages of different domains, which will have to be updated frequently. This process of updating require an effective algorithm to maintain the efficiency of the system.

\section{ACKNOWLEDGMENTS}

My thanks to the experts who have contributed towards development of the work.

\section{REFERENCES}

[1] Bowman, Chelsea Hicks, Matthew Scheffer, Anne H.H. Ngu, Quan Z. Sheng," Discovery and Cataloging of Deep Web Sources” IEEE IRI 2012, August 8-10, 2012.

[2] Y. Li, Y. Wang and J. Du, "E-FFC: an enhanced formfocused crawler for domain-specific deep web databases," Published in Journal of Intelligent Information Systems, Springer, pp.1-26, 2012.

[3] Q. Huanga, Q. Li, H. Li and Z. Yan, "An Approach to incrementaldeep Web Crawling Based on Incremental Harvest Model,"Published in International Workshop on Information andelectronics Engineering, Elsevier Ltd., pp. 1081-1087, 2011.

[4] K. K. Bhatia, A.K. Sharma and R. Madaan. "AKSHR: A novelframework for a Domain-specific Hidden Web Crawler," inproceedings of the 1st International Conference on Parallel,Distributed and Grid Computing (PDGC), IEEE, pp. 307-312,2010.

[5] J. Madhavan, D. Ko, L. Kot, V. Ganapathy, A. Rasmussen and A.Halevy, "Google's Deep Web Crawl," In Proceedings of verylarge Data Bases (VLDB) Endowment, ACM, pp. 1241-1252,2008.

[6] Sergio Flesca, eliomasciari, and Andrea Tagarelli,"A Fuzzy Logic Approach To Wrapping Pdf Documents" Ieee Transactions On Knowledge And Data Engineering, VOL. 23, NO. 12, DECEMBER 2011.

[7] Jer Lang Hong," Data Extraction for Hidden Web Using wordnet" IEEE Transactions On Systems, Man, And Cybernetics-Part C: Applications And Reviews, vol. 41, no. 6, november 2011.

[8] Gang Liu, Kai Liu, Yuan-yuan Dang," Research on discovering Hidden web entries Based ontopic crawling and ontology" 978-1-4244-8165-1/11/-2011 IEEE.

[9] Barbosa, L., Nguyen, H., Nguyen, T., Pinnamaneni, R., Freire, J.: Creating and exploring web form repositories. In: Proceedings of the 2010 international conference on 
Management of data. Pp. 1175-1178. SIGMOD '10, ACM, New York, NY, USA (2010), http://doi.acm.org/ 10.1145/1807167.1807311.

[10] Nan Zhang and Gautam Das. Exploration of hidden web repositories. PVLDB, 4(12):1506\{1507, 2011.

[11] UC Berkeley. Invisible or Deep Web: What it is, Why it exists, How to find it, and Its inherent ambiguity. Available

at http://www.lib.berkeley.edu/teachinglib/Guides/Internet/ invisibleweb.html, July 2006.

[12] Tantan Liu and Gagan Agrawal, "Stratified K-means Clustering Over A Deep Web Data Source” KDD'12, August 12-16, 2012, Beijing, China.
[13] Ritu Khare Yuan An Il-Yeol Song"Understanding Deep Web Search Interfaces" SIGMOD Record, March 2010 (Vol. 39, No. 1).

[14] Fajar Ardian, Sourav S Bhowmick," Efficient Maintenance of Common Keys in Archives of Continuous Query Results from Deep Websites" 978-14244-8960-2/11/- 2011 IEEE

[15] Tim Furche, Georg Gottlob, Giovanni Grasso, Xiaonan Guo, Giorgio Orsi, Christian Schallhart "Automated Form Understanding for the Deep Web" WWW 2012, April 16-20, 2012, Lyon, France.

[16] Radhouane Boughammoura, Lobna Hlaoua, Mohamed Nazih Omri "'Information Technology and e-Services (icites), 2012 International Conference IEEE- 24-26 March 2012. 